\title{
Disclination Dipoles as the Basic Structural Elements of Dielectric Glasses
}

\author{
V.A. Osipov and S.E. Krasavin \\ Joint Institute for Nuclear Research, \\ Bogoliubov Laboratory of Theoretical Physics \\ 141980 Dubna, Moscow region, Russia
}

(October 16, 2018)

\begin{abstract}
We show that the experimentally observed behavior of thermal conductivity of dielectric glasses over a wide temperature range can be explained by a combination of two scattering processes. The first one comes from the phonon scattering due to biaxial dipoles of wedge disclinations while the second one is the Rayleigh type scattering. The results obtained support the cluster picture suggested earlier for glassy materials.
\end{abstract}

Physics of glassy systems has been attracting a considerable interest for a long period. Since the discovery of the anomalous low-temperature behavior of amorphous dielectrics [1] there were many attempts to explain this unusual phenomenon. And yet, in 1986 Freeman and Anderson [2] reviewing both numerous empirical results and theoretical models made a rather drastic conclusion that the thermal conductivity of amorphous dielectrics "is not understood in any temperature range". While at first glance this conclusion looks too pessimistic, it reflects an actual situation existed in glassy physics at that time. It has been found experimentally that the low-temperature anomaly in thermal conductivity, $\kappa$, observed by Zeller and Pohl for some noncrystalline solids [1] is universal. In particular, a $T^{2}$ dependence of $\kappa$ below $1 K$ together with the following characteristic plateau region have been found in the most of glassy materials (see, e.g., review [3]) as well as in some quasicrystalline alloys [ $[$. This finding allows to assume that a realistic theoretical model for description of the thermal conductivity should capture the very essence of these materials, their microstructure. Thus the conclusion of Freeman and Anderson actually suggests that this problem still remains to be solved.

The most widely used model which explains glassy physics below $1 K$ has been proposed by Phillips $[5]$ and Anderson, Halperin, and Varma [6]. In this temperature region, thermal transport takes place via propagating acoustic modes. Thus, $\kappa$ depends on the scattering of the phonons by the structure. Within the model [5, 6, the principal scatterers were proposed to be the tunneling states (TS) in the glass. However, in spite of the success achieved in interpreting experimental data, some important questions were left unanswered. The main of them concern the nature of TS and the source of their universality (see, e.g., discussion in [3:7]).

Since 1972 there have been many attempts to explain the experimental data in terms of propagating thermal phonons scattered by TS [3, 8 11] (in different modifications), density fluctuations (Rayleigh scattering) 1, 3, 10, 12, 14, and the like. Some papers invoked nonpropagating vibrational modes as well [3]. The most successful approach includes a combination of TS and the Rayleigh scattering [10]. Notice that there were attempts to go beyond the TS approximation (see Ref. [15] and the references therein) as well as to do without TS at all. For example, an interesting phenomenological approach was discussed in Ref. [3] where a combination of the structure scattering together with a constant free path was shown to give a good fit to the thermal conductivity data in amorphous arsenic (a-As). As a result, it was suggested that no tunneling state scattering is needed to explain the temperature dependence of $\kappa$ in this material.

Of our special interest is the model proposed by J.C. Phillips [16], who suggested that glasses have cluster structures. It has long been known that there is a close connection between grain boundaries in materials with pronounced cluster structure and wedge disclination dipoles (WDD) 17]. Recently, this analogy has been successfully applied for description of the old experiments in polycrystals on the basis of the WDD-induced scattering [18]. As was mentioned in [3], the scattering in polycrystals has a close parallel in amorphous solids.

Another approach which takes into account topological defects in glasses came from geometrical and topological considerations. For example, Kléman (see review [19]) proposed that disordered covalent systems can be described in terms of distributions of defects, most probably disclinations, in a crystal situated in a space of constant curvature. This idea was explored by Nelson 20] in description of metal glasses. Similarly, Duffy and Rivier 21] considered a continuous random network formed by 5 and/or 7 rings in the hexagonal lattice. In this case one can also expect the appearance of 5-7 disclination pairs (dipoles of disclinations). Let us mention also papers [22] where elastic dipoles have been introduced within the glassy model.

In this Letter, we present a new look at the problem of thermal conductivity in dielectric glasses as well as at their microstructure. Actually, we reexamine the old ideas of the previous two paragraphs from another point of view. The model proposed here was inspired by a recent finding that a biaxial WDD is a rather unique scatterer 18. Namely, it has been shown that the mean free path $l_{D}$ of thermal phonons scattered by biaxial WDD with nonskew axes of rotation behaves like $l_{D} \sim q^{-1}$ at small $q$ while $l_{D} \rightarrow$ const with $q$ increasing. As is 
known, a similar behavior, but with $l_{q} \sim q^{-2}$ for small $q$, has been found in old experiments on glasses [23]. However, the later experiments showed that in fact $l_{q} \sim q^{-1}$ in agreement with the behavior of $l_{D}$. The main aim of this paper is to show that the phonon scattering due to biaxial WDD, being combined with the Rayleigh scattering, can explain the thermal conductivity experiments in amorphous dielectrics over a wide temperature range. As an example, we compare the results obtained from the experimental data for vitreous silica $\left(\mathrm{a}-\mathrm{SiO}_{2}\right)$. Moreover, considering WDD as the basic structural elements of glasses one can explain the universal behavior seen in different amorphous dielectrics.

To gain a better understanding, let us discuss briefly the generally accepted approaches as well as the main problems pointed out in [2]. The starting point for the calculations is the known kinetic formula

$$
\kappa=\frac{1}{3} \int_{0}^{\omega_{D}} C(\omega, T) v l(\omega, T) d \omega,
$$

where $C(\omega, T) d \omega$ is the specific heat contributed by phonons within the frequency interval $d \omega, v$ is an average phonon velocity, and $l(\omega, T)$ is the mean free path of a phonon. As usual, at low temperatures we can assume a constant $v$ and a density of phonon states quadratic in $\omega$ with the Debye cutoff $\omega_{D}$. To reproduce the observed $\kappa$ over the full temperature range the best form of the total mean free path was taken to be 10

$$
l(\omega, T)=\left(l_{t}^{-1}+l_{R}^{-1}\right)^{-1}+l_{\text {min }},
$$

where $l_{t}$ is the mean free path due to scattering from TS's, $l_{R}$ comes from the Rayleigh scattering, and $l_{\text {min }}$ is constant which must be included to prevent $l$ from becoming unphysically small. It should be stressed that it is this required cut-off $l_{\min }$ that causes the main problem. In fact, it has no natural explanation. A different situation arises in a- $A s$ where the experimental data were described by a combination of a constant mean free path resulting from phonon scattering by small holes in samples and the Rayleigh type scattering. In this case, the Rayleigh scattering becomes important in the highfrequency limit. Thus, one has to use the interpolation formula which describes the Rayleigh scattering over the complete frequency range. It reads [3]

$$
l_{R}=D^{-1}\left(\frac{\hbar \omega}{k_{B}}\right)^{-4}+l_{0},
$$

where $\mathrm{D}$ is a constant which has been considered as a fitting parameter, and $k_{B}$ is the Boltzman constant. This approach explains the thermal conductivity over the full temperature range without the TS scattering. However, this model is restricted to description of a- $A s$ where $\kappa \sim$ $T^{3}$ has been observed below $0.5 K$ instead of $\kappa \sim T^{2}$ for the most of glasses.

Let us return to the paper [2] where the following three characteristic temperature regions have been distinguished:
1. At $T / \Theta \leq 0.01$ the phonon scattering is such that $l / \lambda \approx 150$ for any type of glass. Here $\Theta$ is the Debye temperature, and $\lambda$ is the wavelength of a phonon. The main question is why this relation is valid for such a varied spectrum of glasses?

2. In the plateau region, $0.01 \leq T / \Theta \leq 0.1$, the mean free path of the phonons rapidly decreases with increasing $q$. What is the scattering mechanism responsible for this frequency dependence?

3. At $T / \Theta \geq 0.1$ it is not even clear what excitations are responsible for thermal transport.

We will try to answer some of these questions below within our model. First of all, let us reproduce briefly the main results of the previous paper where the scattering of thermal phonons by WDD was studied [18]. For simplicity, in what follows we consider only biaxial WDD with nonskew axes of rotation (below the term "biaxial WDD" will refer only to this type of biaxial WDD).

As is known, dislocations and disclinations cause additional strain fields which lead to the scattering of phonons. A mean free path of phonons of frequency $\omega$ scattered by the potential associated with a static deformation of a lattice caused by straight WDD can be calculated within the generally accepted approach (see., e.g. [24,25]). An effective perturbation energy due to the strain field caused by a single WDD is written as $U(\vec{r})=\hbar \omega \gamma S p E_{A B}$, where $\hbar \omega$ is the phonon energy with the wavevector $\vec{q}, \omega=q v, \gamma$ is the Grüneisen constant, and $E_{A B}$ is the strain tensor due to WDD. For a biaxial WDD, the strain matrix is found to have a complex form. To simplify the problem, we assume that incident phonons lie normally to disclination lines and choose the suitable geometry with disclination lines directed along the $z$-axis and the dipole's arm oriented along the $x$-axis. In this case, $U(\vec{r})$ takes the form:

$$
U(x, y)=\frac{B}{2} \ln \frac{(x+L)^{2}+y^{2}}{(x-L)^{2}+y^{2}}
$$

where $B=\hbar q v \gamma \nu(1-2 \sigma) /(1-\sigma), \nu$ is the Frank index, $\sigma$ is the Poisson constant, and $2 L$ is the dipole separation.

In accordance with Eq.(4), the problem reduces to the two-dimensional case with the phonon mean free path given by

$$
l_{q}^{-1}=n_{i} \int_{0}^{2 \pi}(1-\cos \theta) \Re(\theta) d \theta,
$$

where $\Re(\theta)$ is an effective differential scattering radius, and $n_{i}$ is the areal density of defects. Within the Born approximation, $\Re(\theta)$ is determined as 25.

$$
\Re(\theta)=\frac{q S^{2}}{2 \pi \hbar^{2} v^{2}} \overline{|<\vec{q}| U(\vec{r})\left|\vec{q}^{\prime}>\right|^{2}},
$$

where all vectors are two-dimensional ones, $\vec{p}=\vec{q}-\vec{q}^{\prime}$, $S$ is a projected area, and the bar denotes an averaging procedure over $\alpha$ which defines an angle between $\vec{p}$ and the $x$-axis. In other words, it means the averaging over 
randomly oriented dipoles in the $x y$ plane. Evidently, the problem reduces to the estimation of the matrix element in Eq.(6) with the potential from Eq.(4). The result is

$$
U(p, \alpha)=-\frac{4 \pi i B}{p^{2}} \sin (p L \cos \alpha) .
$$

After averaging of $|U(p, \alpha)|^{2}$ over $\alpha$ and following integration in Eq.(5) with respect to $\theta$ one obtains

$$
\begin{array}{r}
l_{D}^{-1}=8 A^{2}(\nu L)^{2} n_{i} q\left(J_{0}^{2}(2 q L)+J_{1}^{2}(2 q L)-\right. \\
\left.\frac{1}{2 q L} J_{0}(2 q L) J_{1}(2 q L)\right),
\end{array}
$$

where $A=\pi \gamma(1-2 \sigma) /(1-\sigma)$, and $J_{n}(t)$ are the Bessel functions. Notice that the behavior of $l_{D}$ in Eq.(8) is actually governed by the only parameter $2 L$ which characterizes the dipole separation. In the long wavelength limit one gets $l_{D} \sim q^{-1}$ while for $\lambda<L$ we obtain that $l_{D} \rightarrow$ const. It should be stressed that the appearance of the $q$-independent region distinguishes remarkably this scatterer from the others known.

The total phonon mean free path is obtained by combining the inverse lengths

$$
l_{\text {tot }}(\omega)=\left(l_{R}^{-1}+l_{D}^{-1}\right)^{-1} .
$$

with $l_{R}$ and $l_{D}$ from Eq.(2) and Eq.(8), respectively. An important consequence of Eq.(9) is that we do not need any cut-off procedure in calculating $\kappa$.

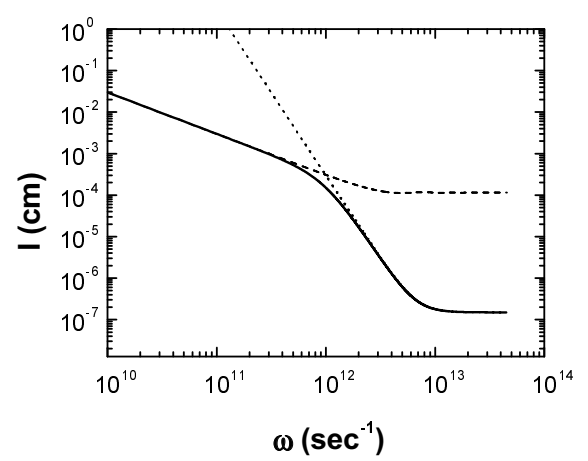

FIG. 1. Phonon mean free paths $l_{D}$ (dashed line), $l_{R}$ (dotted line), and $l_{\text {tot }}$ (solid line) as functions of frequency. The parameter set used is: $n_{i}=5 \times 10^{11} \mathrm{~cm}^{-2}, 2 L=20 \AA$, $\nu=0.1, v=4.1 \times 10^{5} \mathrm{~cm} / \mathrm{sec}, A=2.6, D=1.0 \mathrm{~cm}^{-1} K^{-4}$, and $l_{0}=15 \AA$.

Fig.1 shows $l_{D}, l_{R}$, and $l_{\text {tot }}$ for a characteristic set of the model parameters. Notice that the parameters for the Rayleigh scattering are chosen to be in agreement with those for a-SiO $\mathrm{S}_{2}[3]$. One can clearly see that at low frequencies the dominant scattering mechanism comes from the WDD-induced scattering, then there appears a region with the two scattering processes involved, and finally the Rayleigh scattering becomes dominant at high frequencies. This specific behavior results from the fact that both $l_{R}$ and $l_{D}$ tend to constants when $q$ increases. Obviously, any $q$ dependence of scatterers would break this picture at large $q$. The main parameters which actually define $l_{\text {tot }}$ are $D$ from the Rayleigh scattering and $2 L$ from the WDD scattering.

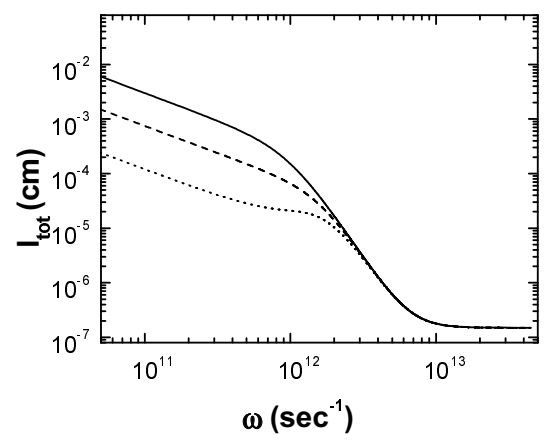

FIG. 2. Phonon mean free path $l_{\text {tot }}$ as a function of frequency at $2 L=20 \AA$ (solid line), $2 L=40 \AA$ (dashed line), and $2 L=100 \AA$ (dotted line). The parameter set is the same as in Fig.1.

As an example, Fig.2 shows $l_{\text {tot }}$ for different dipole separations. It is interesting to note that the size of the transition region decreases remarkably with $2 L$ increasing. Notice that this region alone is responsible for the plateau in the thermal conductivity.

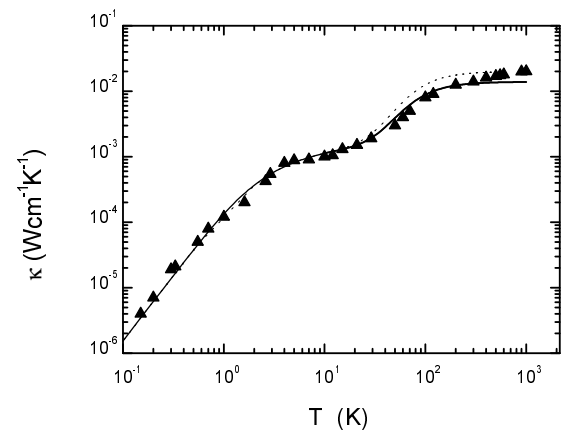

FIG. 3. Thermal conductivity vs temperature calculated according to Eq.(10) with $l_{\text {tot }}$ from Eq.(9) at $l_{0}=10 \AA$ (solid line) and $l_{0}=15 \AA$ (dotted line). The parameter set is the same as in Fig. $1, \Theta=342 \mathrm{~K}$. Experimental data for a- $\mathrm{SiO}_{2}$ from Ref.[1] are indicated by triangles. 
The last step is to calculate $\kappa$ in Eq.(1) with $l_{\text {tot }}$. For this purpose, it is convenient to rewrite Eq.(1) in the dimensionless form

$$
\kappa=\frac{k_{B}^{4} T^{3}}{2 \pi^{2} \hbar^{3} v^{2}} \int_{0}^{\Theta / T} x^{4} e^{x}\left(e^{x}-1\right)^{-2} l_{t o t}(x) d x,
$$

where $x=\hbar \omega / k_{B} T$, and the specific heat capacity is chosen in the standard Debye form. The result is shown in Fig. 3 where the experimental points for a-SiO $\mathrm{S}_{2}$ are marked by triangles. As is seen, there is a good agreement over a wide temperature range. For $l_{0}=15 \AA$ some deviations from the experimental data appear above $40 \mathrm{~K}$ but the correct qualitative behavior retains even at high temperatures. The best fit for a- $\mathrm{SiO}_{2}$ is obtained for $2 L=20 \AA$. Notice that this value agrees with a cluster size expected in vitreous silica [16]. Let us stress once more that we have no need for poorly defined parameters like the cut-off $l_{\text {min }}$ in Eq.(2) or an additional to $l_{R}$ constant free path proposed in [3] to fit the data below $0.5 K$.

Now we can try to answer the questions formulated in the beginning of this paper. In accordance with Fig.1, at low frequencies the dominant contribution comes from $l_{D}$. One can easily estimate the value of $l_{D} / \lambda$ in this region. Spreading out Eq.(8) at $q L \ll 1$ one gets

$$
\frac{l_{D}}{\lambda}=\frac{1}{8 \pi A^{2}(\nu L)^{2} n_{i}} .
$$

This is the constant which depends on the model parameters. Thus, in accordance with our model, the only parameters which determine the behavior of glasses at low temperatures come from their microstructure which includes biaxial WDD as the basic elements. The main parameters are the density of WDD, their Frank index, and the dipole separation. In particular, for our choice of parameters, $l_{D} / \lambda \sim 135$. Let us emphasize that the experimental data for various glasses given in [1] can be described by minor variations of these main parameters (together with $D$ ). Thus the relation $l_{\text {tot }} / \lambda \sim 150$ is indeed satisfied for all these dielectric glasses. Based on the results of our analysis, we can conclude that:

(i) the WDD-induced scattering of phonons can explain the established relation $l / \lambda \sim 150$ for various glasses;

(ii) in the plateau region both mechanisms (WDDinduced and the Rayleigh scattering) should be accounted for to produce a necessary behavior;

(iii) with increasing temperature, the Rayleigh scattering becomes dominant. While there are some deviations from the experimental data at high temperatures, the general behavior looks quite correct. Thus nothing else but phonons transport the heat at $T>0.1 \Theta$, and no any other excitations are needed.

To summarize, we have presented a new look at the problem of the thermal conductivity in amorphous dielectrics. The main suggestion is based on the introduction of biaxial WDD as the basic structural elements of glasses. The unique properties of the WDD-induced phonon scattering enabled us to describe the universal behavior of the thermal conductivity of amorphous dielectrics over a wide temperature range. Thus, the results obtained support the cluster picture of amorphous dielectrics suggested by Phillips [16] and shed some light on the relationship between the microstructure of glasses and their transport properties.

This work has been supported by the Russian Foundation for Basic Research under grant No. 97-02-16623.

[1] R.C. Zeller and R.O. Pohl, Phys. Rev. B 4 (1971) 2029.

[2] J.J. Freeman and A.C. Anderson, Phys. Rev. B 34 (1986) 5684.

[3] D.P. Jones, N. Thomas, and W.A. Phillips, Phil. Mag. B 38 (1978) 271.

[4] M.A. Chernikov, A. Bianchi, and H.R. Ott, Phys. Rev. B 51 (1995) 153.

[5] W.A. Phillips, J.Low Temp. Phys. 7 (1972) 351.

[6] P.W. Anderson, B.I. Halperin, and C.M. Varma, Phil. Mag. 25 (1972) 1.

[7] Yu.M. Galperin, V.G. Karpov, and V.I. Kozub, Adv. Phys. 38 (1989) 669.

[8] M.P. Zaitlin and A.C. Anderson, Phys. Rev. B 12 (1975) 4475.

[9] T.L. Smith, P.J. Anthony, and A.C. Anderson, Phys. Rev. B 17 (1978) 4997.

[10] D.P. Jones and W.A. Phillips, Phys. Rev. B 27 (1983) 3891.

[11] A.J. Leadbetter, A.P. Jeapes, C.G. Waterfield, and R. Maynard, J. Phys. (Paris) 38 (1977) 95.

[12] G.J. Morgan and D. Smith, J. Phys. C 7 (1974) 649.

[13] D. Walton, Phys. Rev. B 16 (1977) 3723.

[14] J.E. Graebner, B. Golding, and L.C. Allen, Phys. Rev. B 34 (1986) 5696.

[15] R. Kühn and U. Horstmann, Phys. Rev. Lett. 78 (1997) 4067.

[16] J.C. Phillips, Phys. Rev. B 24 (1981) 1744; ibid. 25 (1981) 1397.

[17] J.C.M. Li, Surf. Sci. 31 (1972) 12.

[18] V.A. Osipov and S.E. Krasavin, J. Phys.: Cond. Matt. 10 (1998) L639.

[19] M. Kléman, Adv. Phys. 38 (1989) 605.

[20] D.R. Nelson, Phys. Rev. B 28 (1983) 5515.

[21] D.N. Duffy and N. Rivier, J. Phys. (Paris) 43 (1982) 293.

[22] E.R. Grannan, M.Randeria, and J.P. Sethna, Phys. Rev. B 41 (1990) 7784, 7799.

[23] R. Berman, Phys. Rev. 76 (1949) 315.

[24] P.G. Klemens, Proc. Phys. Soc. Lond. A 68 (1955) 1113.

[25] J.M. Ziman, Electrons and Phonons: The Theory of Transport Phenomena in Solids (Clarendon, Oxford, 1960). 